\title{
PROJECTIVE LATTICES AND BOUNDED HOMOMORPHISMS
}

\author{
Alan Kostinsky
}

\begin{abstract}
The main purpose of this paper is to prove that a finitely generated lattice is projective iff it is imbeddable in a free lattice. This result appears as a consequence of a more general theorem, in which a sufficient condition for projectivity is given in terms of the notion (due to Ralph McKenzie) of bounded homomorphism.
\end{abstract}

In [1, Theorems 4.1, 4.4] Baker and Hales completely describe the distributive projective lattices and obtain as a corollary the fact that a finite distributive lattice is projective iff it is imbeddable in a free lattice. This last result has been improved by McKenzie, who finds in [6, proof of Theorem 6.3] that for any finite lattice $L, L$ is projective iff it is imbeddable in a free lattice. McKenzie's proof uses some ideas due to B. Jónsson. To extend the theorem to finitely generated lattices we sharpan arguments of [6]. As stated above, we use the notion of bounded homomorphism; this idea is defined by McKenzie in [6], and it plays an important role in that paper. Theorem 3.4 below was first announced in the author's abstract [4].

1. Preliminaries. We regard a lattice as an algebraic structure $\langle L,+, \cdot\rangle$ in which the sum (join) and product (meet) satisfy the usual equational axioms. It will not cause confusion to refer to a lattice by naming its universe. We denote by $\leqq$ the ordering of the lattice $L$, that is, the partial ordering naturally associated with $L(x<y$ means $x \leqq y$ and $x \neq y)$. If the greatest lower bound (least upper bound) of a subset $U$ of $L$ exists in $L$, it is denoted $\Lambda U(\Lambda U)$.

The notation and terminology used for maps is largely standard. By an epimorphism of a lattice $L$ into a lattice $M$ we mean a homomorphism of $L$ onto $M$. For any $\operatorname{sets} L, M, N$, and any maps $f: L \rightarrow M$ and $g: M \rightarrow N$, the composite map (of $L$ into $N$ ) is denoted $g \circ f$. (In the arrow notation, maps-whether homomorphisms or notwhich are onto may be indicated by the use of a double-headed arrow, $\rightarrow$.)

A chain is a lattice whose ordering is a linear ordering. A chain is bounded iff it has a least element and a greatest element. Any ordinal $\alpha$ may be viewed as the chain whose ordering is the natural ordering of $\alpha$. The set of all natural numbers is denoted $\omega$.

We regard Boolean algebras as lattices (thus, zero, one, and complementation are not primitive operations). 
The terms of the language of lattice theory are built up from individual variables $v_{0}, v_{1}, \cdots$ and the binary operation symbols $\vee$ and $\wedge$ (interpreted in lattices as + and $\cdot$, respectively). The notion of length of a term is assumed familiar. Let $\tau$ be a term of lattice theory with variables among $v_{0}, \cdots, v_{n}$; let $L$ be a lattice and $x_{0}, \cdots, x_{n} \in L$. Then by $\tau\left[L, x_{0}, \cdots, x_{n}\right]$ we mean the denotation of $\tau$ in $L$ under the assignment $v_{i} \rightarrow x_{i}(i \leqq n)$. If $\tau$ is a term of lattice theory, $L$ and $M$ are lattices, $x_{0}, \cdots, x_{n} \in L$, and $f$ is a homomorphism of $L$ into $M$, then $f\left(\tau\left[L, x_{0}, \cdots, x_{n}\right]\right)=\tau\left[M, f x_{0}, \cdots, f x_{n}\right]$.

The free lattices are especially important for our present work. For any nonempty set $X$, we let $F L(X)$ denote some fixed lattice freely generated by $X$. Recall that if $X_{0}, X_{1}$ are disjoint nonempty subsets of $X$, and if $x_{i}$ is in the sublattice of $F L(X)$ generated by $X_{i}(i=0,1)$, then $x_{0} \cdot x_{1}<x_{0}<x_{0}+x_{1}$ in $F L(X)$.

Whitman's famous solution to the word problem for lattices, in [7], provides a characterization of the free lattice as follows. Suppose $L$ is a lattice generated by $X \neq \varnothing$. Then $L$ is freely generated by $X$ (that is, $L \cong F L(X)$ ) iff all of the following hold in $L$ : (W0) for all $x, x^{\prime} \in X$, if $x \leqq x^{\prime}$ then $x=x^{\prime}$; (W1) for all $x \in X$ and all $a, b \in L$, if $a \cdot b \leqq x$ then $a \leqq x$ or $b \leqq x$, and if $x \leqq a+b$ then $x \leqq a$ or $x \leqq b$; (W2) for all $a, b, c, d \in L$, if $a \cdot b \leqq c+d$ then $a \leqq c+d$ or $b \leqq c+d$ or $a \cdot b \leqq c$ or $a \cdot b \leqq d$. The latter two properties (which we refer to as Whitman's (W1) and (W2)) are frequently used below. Note that (W2) makes no reference to a generating set; for any lattice $L$, there is no ambiguity in saying that Whitman's (W2) holds (or does not hold) in $L$.

We also use the following theorem, derived by Jónsson from a result of Whitman [7]:

THEOREM 1.1. (Jónsson [3, Lemma 2.6, p. 262]) In any free lattice $F L(X)$, the following hold: for all $u, a, b, c \in F L(X)$, if $u=a \cdot b=a \cdot c$ then $u=a \cdot(b+c)$; if $u=a+b=a+c$ then $u=a+b \cdot c$.

Finally we recall some basic facts about linear sums of lattices. Let $\langle E, \leqq\rangle$ be a linearly ordered structure and let $\left\langle L_{e}: e \in E\right\rangle$ be a system of lattices such that $e \neq e^{\prime}$ implies $L_{e} \cap L_{e^{\prime}}=\varnothing$. Then the linear sum $\Sigma_{E} L_{e}$ is the lattice $L$ completely determined by the following: $L=\bigcup\left\{L_{e}: e \in E\right\}$; for each $e \in E, L_{e}$ is a sublattice of $L$; and whenever $e \neq e^{\prime}, x \in L_{e}, y \in L_{e^{\prime}}$, then $x<y$ in $L$ iff $e<e^{\prime}$ in $E$. Roughly speaking, $L$ is constructed simply by stacking up the $L_{e}$ in accordance with the ordering of $E$.

A lattice is linearly indecomposable iff it is not the linear sum of two lattices. For any lattice $L$ there are a linearly ordered struc- 
ture $E$ (unique up to isomorphism) and a system of lattices $\left\langle L_{e}\right.$ : $e \in E>$ (with range $\left\{L_{e}: e \in E\right\}$ uniquely determined by $L$ ) such that each $L_{e}$ is linearly indecomposable and $L=\Sigma_{E} L_{e}$; the $L_{e}$ are called the linear components of $L$.

2. Projectivity and bounded homomorphisms.

Definition 2.1. A lattice $L$ is projective (in the category of all lattices and lattice homomorphisms) iff for any lattices $M, N$, and any lattice homomorphisms $h: L \rightarrow N$ and $g: M \rightarrow N$ ( $g$ onto), there is a homomorphism $f: L \rightarrow M$ such that $g \circ f=h$.

It is well-known that there are simpler descriptions of projectivity than 2.1; in particular, we have:

Note 2.2. For any lattice $L$ the following three conditions are equivalent:

(1) $L$ is projective;

(2) for any lattice $M$ and any epimorphism $f: M \rightarrow L$, there is a homomorphism $g: L \rightarrow M$ such that $f \circ g$ is the identity map on $L$;

(3) there are a free lattice $F L(X)$, an epimorphism $f: F L(X) \rightarrow$ $L$, and a homomorphism $g: L \rightarrow F L(X)$ such that $f \circ g$ is the identity map on $L$.

We shall use formulation (3) in this paper; (2) is used in [1].

Note that every projective lattice is isomorphic to a sublattice of a free lattice (the map $g$ of (3) clearly must be one-to-one). Also, every free lattice is obviously projective. Baker and Hales [1, Theorem 3.1, p. 473] prove that a countable lattice is projective iff each of its linear components is projective.

In [2, Theorem 6, p, 271] Galvin and Jónsson show that a distributive lattice $L$ is imbeddable in a free lattice iff $L$ is countable and each linear component of $L$ is one of the following: a one-element lattice, an eight-element Boolean algebra, or an isomorphic image of the direct product of a countable chain and a two-element chain. Using this result, Baker and Hales [1, Theorem 4.1, p. 474] characterize the distributive projective lattices as follows: a distributive lattice $L$ is projective iff $L$ is countable and each linear component of $L$ is one of the following: a one-element lattice, an eight-element Boolean algebra, or an isomorphic image of the direct product of a countable bounded chain and a two-element chain.

As to non-projective lattices, the above remarks readily yield many examples. Thus, all the non-distributive modular lattices are non-projective ${ }^{1}$, for they are not imbeddable in free lattices (recall

1 This fact was pointed out by the referee. 
that the five-element lattice with three mutually incomparable elements is imbeddable in every non-distributive modular lattice; apply Jónsson's Theorem 1.1). And, as observed in [1], the above-mentioned results on distributive lattices show that the direct product $2 \times \omega$ is a distributive non-projective lattice imbeddable in $F L(\omega)$ (and hence imbeddable in $F L(3)$, by Whitman [8, Theorem 6, p. 109]).

Definition 2.3 (McKenzie [6, Definition 5.2]) Suppose $L, M$ are lattices and $f$ is a homomorphism of $L$ into $M$. We say $f$ is upper bounded iff for each $b \in M,\{a \in L: f a \leqq b\}$ either is empty or has a greatest element; $f$ is lower bounded iff for each $b \in M,\{a \in L: b \leqq f a\}$ either is empty or has a least element. We say $f$ is bounded iff it is both upper and lower bounded.

N. B. These notions are defined with respect to the entire codomain $M$ of $f$, not merely with respect to the range of $f$. The intended codomain will be specified below in the rare cases where there is ambiguity.

Note 2.4. Suppose $L, M$ are lattices and $f$ is a homomorphism of $L$ into $M$; suppose that $f$, viewed as a homomorphism of $L$ into $M$, is bounded. Then if $N$ is any sublattice of $M$ which includes the range of $f, f$ is also bounded as a homomorphism of $L$ into $N$. (Trivial.)

Usually we shall deal with epimorphisms. If $f$ is an epimorphism of $L$ into $M$, clearly $f$ is uppar iff for each $b \in M,\{a \in L: f a=b\}$ has a greatest element; similarly for the other two notions of 2.3 .

Definition 2.5. Let be the class of all lattices which are bounded epimorphic images of free lattices. $(L \in \mathscr{B}$ iff there are a free lattice $F L(X)$ and an epimorphism $f: F L(X) \rightarrow L$ such that $f$ is bounded.)

Trivially every free lattice is in $\mathscr{B}$. It follows readily from McKenzie [6, Lemma 5.2] that every finitely generated sublattice of a free lattice is in $\mathscr{B}$ (see also the proof of Theorem 3.4 below).

An element of $\mathscr{\mathscr { B }}$ need not be imbeddable in a free lattice. Thus, according to [6, remarks following Theorem 5.1], every finite Boolean algebra is in $\mathscr{B}$; but, by the Galvin-Jónsson result stated above, a Boolean algebra with more than eight elements is not imbeddable in a free lattice. Those elements of $\mathscr{B}$ which are not imbeddable in free lattices are, of course, not projective; we shall see that all other elements of $\mathscr{B}$ are projective (Theorem 3.3).

In Corollary 5.3 of [6], McKenzie shows that the two properties 
of free lattices described in Jónsson's Theorem 1.1 carry over to the lattices of $\mathscr{S}$; it follows that every non-distributive modular lattice fails to be in $\mathscr{B}$.

Recall from above that Baker and Hales have shown that if $L$ is countable and is a linear sum of projective lattices, then $L$ is projective. The situation is quite different for $\mathscr{B}$. as we shall see in Lemma 2.7. For the moment we remark that every countably infinite chain is projective, but is not in 8 .

Lemмa 2.6. Suppose $f$ is a homomorphism of a free lattice $F L(X)$ into a lattice $L$. Then the following hold:

(1) if $f$ is upper bounded then for each $b \in L,\{x \in X: f x \leqq b\}$ is finite;

(2) if $f$ is lower bounded then for each $b \in L,\{x \in X: b \leqq f x\}$ is finite.

Proof. We prove (1) ((2) is similar). Suppose $\{x \in X: f x \leqq b\}$ is infinite. Let $z$ be any element of $\{a \in F L(X): f a \leqq b\}$; let $X^{\prime}$ be a finite subset of $X$ such that $z$ is in the sublattice of $F L(X)$ generated by $X^{\prime}$; and choose $x_{0} \in\{x \in X: f x \leqq b\} \sim X^{\prime}$. Then $f\left(z+x_{0}\right) \leqq b$, and $z<z+x_{0}$ in $F L(X)$ (see $\S 1$ ). Thus $f$ is not upper bounded.

LEMmA 2.7. Suppose $L=\Sigma_{E} L_{e}$, where $E$ is any infinite linearly ordered structure and the $L_{e}$ are any lattices. Then $L \notin \mathscr{B}$.

Proof. Say $f: F L(X) \rightarrow L$. We show that $f$ cannot be bounded. For every nonempty subset $S$ of $E, \cup\left\{L_{e}: e \in S\right\}$ is a sublattice of $L$; hence $f$ must map an element of $X$ into every $L_{e}$. For each $e \in E$, choose $x_{e} \in X$ such that $f x_{e} \in L_{e}$, The $x_{e}$ are distinct. Let $d \in E$. For each $e \in E, f x_{e}$ is comparable to $f x_{d}$ in $L$; hence either $\left\{x \in X: f x \leqq f x_{d}\right\}$ or $\left\{x \in X: f x_{d} \leqq f x\right\}$ is infinite. Thus, by 2.3 and 2.6, $f$ is not bounded.

3. Main results. Lemma 3.1 and Theorem 3.3 below are closely based on Lemma 5.2 of McKenzie's paper [6]; 3.1 generalizes that lemma.

LEMMA 3.1. Suppose $L$ is a lattice generated (not necessarily freely) by a set $X$, and suppose $f$ is a homomorphism of $L$ into a free lattice $F L(Y)$. Then the following hold:

(1) if for each $b \in F L(Y),\{x \in X: f x \leqq b\}$ is finite, then $f$ is upper bounded;

(2) if for each $b \in F L(Y),\{x \in X: b \leqq f x\}$ is finite, then $f$ is lower bounded. 
Proof. We prove (1); a dual argument works for (2). Assume the hypothesis of (1). Let $T$ be the set of all elements $b$ of $F L(Y)$ such that $\{a \in L: f a \leqq b\}$ either is empty or has a greatest element. As in [6, Lemma 5.2], we show inductively that $T=F L(Y)$; we use the fact that Whitman's (W1) and (W2) hold in free lattices (see $\S 1$ ).

First we show that $Y \subseteq T$. Suppose $y \in Y$ and $\{a \in L: f a \leqq y\} \neq$ $\varnothing$. Since $L$ is generated by $X$, repeated application of Whitman's (W1) to a relation $f a \leqq y(a \in L)$ yields an $x \in X$ such that $f x \leqq y$. This, together with the hypothesis of (1), shows that $a_{0}=\mathrm{V}\{x \in X$ : $f x \leqq y\}$ exists in $L$ and that $f a_{0} \leqq y$. We claim that for all $a \in L$, if $f a \leqq y$ then $a \leqq a_{0}$. To see this, let $S$ be the set of $a \in L$ for which the claim is true. Obviously $X \subseteq S$; and if $a, a^{\prime} \in S$ then, trivially, $a+a^{\prime} \in S$. If $a, \alpha^{\prime} \in S$ and $f a \cdot f a^{\prime} \leqq y$, then by Whitman's (W1), $f a \leqq y$ or $f a^{\prime} \leqq y$, so that $a \leqq a_{0}$ or $a^{\prime} \leqq a_{0}$; hence $a \cdot a^{\prime} \leqq a_{0}$. Therefore $S=L$, as claimed. Thus $a_{0}$ is the greatest element of $\{a \in L: f a \leqq y\}$, and $y \in T$.

It is easy to see that $T$ is closed under product. If $b_{0}, b_{1} \in T$ and $\left\{a \in L: f a \leqq b_{0} \cdot b_{1}\right\}$ is nonempty, then both $\left\{a \in L: f a \leqq b_{0}\right\}$ and $\{a \in L$ : $\left.f a \leqq b_{1}\right\}$ are nonempty, hence have largest elements $a_{0}, a_{1}$, respectively. Clearly $a_{0} \cdot a_{1}$ is the largest element of $\left\{a \in L: f a \leqq b_{0} \cdot b_{1}\right\}$.

Finally we show that $T$ is closed under sum. Suppose $b_{0}, b_{1} \in T$ and $\left\{a \in L: f a \leqq b_{0}+b_{1}\right\} \neq \varnothing$. For $i \in\{0,1\}$, in case $\left\{a \in L: f a \leqq b_{i}\right\} \neq$ $\varnothing$, let $a_{i}$ be its largest element. Next consider the set $\{x \in X: f x \leqq$ $\left.b_{0}+b_{1}\right\}$; if this set is empty then there is a term $\tau$ of lattice theory of some minimum length $>1$ such that for some $x_{0}, \cdots, x_{n} \in X$, $f\left(\tau\left[L, x_{0}, \cdots, x_{n}\right]\right) \leqq b_{0}+b_{1} ; \tau$ must have the form $\tau_{0} \wedge \tau_{1}$, so that $\tau_{0}\left[F L(Y), f x_{0}, \cdots, f x_{n}\right] \cdot \tau_{1}\left[F L(Y), f x_{0}, \cdots, f x_{n}\right] \leqq b_{0}+b_{1} ;$ but $\tau_{\jmath}[F L(Y)$, $\left.f x_{0}, \cdots, f x_{n}\right] \geqq b_{0}+b_{1}$ for $j \in\{0,1\}$; hence, by Whitman's (W2) in $F L(Y)$, for $i=0$ or $i=1, f\left(\tau\left[L, x_{0}, \cdots, x_{n}\right]\right) \leqq b_{i}$. Therefore, we see that at least one of the three elements $a_{0}, a_{1}, \bigvee\left\{x \in X: f x \leqq b_{0}+b_{1}\right\}$ is defined in $L$; let $a_{2}$ be the sum of those that are defined.

Clearly $f a_{2} \leqq b_{0}+b_{1}$. Now we claim that for all $a \in L$, if $f a \leqq$ $b_{0}+b_{1}$ then $a \leqq a_{2}$. Let $S$ be the set of $a \in L$ for which this is true. Obviously $X \subseteq S$; and if $a, a^{\prime} \in S$ then $a+a^{\prime} \in S$. If $a, a^{\prime} \in S$ and $f a \cdot f a^{\prime} \leqq b_{0}+b_{1}$, then by Whitman's (W2) in $F L(Y)$, we have at least one of the following: $f a \leqq b_{0}+b_{1}, f a^{\prime} \leqq b_{0}+b_{1}, f a \cdot f a^{\prime} \leqq b_{1}, f a \cdot f a^{\prime} \leqq$ $b_{1}$; in the first case $a \leqq a_{2}$ by assumption $a \in S$, so $a \cdot a^{\prime} \leqq a_{2}$; in the last case $a_{1}$ must be defined and $a \cdot a^{\prime} \leqq a_{1} \leqq a_{2}$; the other cases are similar, so $a \cdot a^{\prime} \in S$. Therefore $S=L$, as claimed. It follows that $b_{0}+b_{1} \in T$.

Thus, $T=F L(Y)$, that is, $f$ is upper bounded.

Corollary 3.2. Suppose $f$ is a homomorphism of a free lattice $F L(X)$ into a free lattice $F L(Y)$. Then $f$ is bounded iff for each 
$b \in F L(Y),\{x \in X: f x$ is comparable to $b\}$ is finite. (Immediate from 2.6 and 3.1.)

Theorem 3.3. Suppose $L \in \mathscr{B}$ and Whitman's (W2) holds in L. Then $L$ is projective.

Proof. We are given $f: F L(X) \rightarrow L, f$ a bounded homomorphism. Define $\alpha$ and $\beta$, maps of $L$ into $F L(X)$, as follows: for each $b \in L$, $\alpha b$ is the greatest element of $\{a \in F L(X): f a \leqq b\}, \beta b$ is the least element of $\{a \in F L(X): f a \leqq b\}$. Certain properties of $\alpha$ and $\beta$ are immediate. Thus, for each $b \in L, f \beta b=f \alpha b=b$ and $\beta b \leqq \alpha b$. Also, $\beta$ preserves sum and $\alpha$ preserves product; that is for any $b_{0}, b_{1} \in L$, $\beta\left(b_{0}+b_{1}\right)=\beta b_{0}+\beta b_{1}$ and $\alpha\left(b_{0} \cdot b_{1}\right)=\alpha b_{0} \cdot \alpha b_{1}$. And both $\alpha$ and $\beta$ are order-preserving.

We claim an additional property for $\beta$. Let $b_{0}, b_{1} \in L$. By Lemma 2.6, the set $S=\left\{x \in X: b_{0} \cdot b_{1} \leqq f x\right\}$ is finite. Our claim is that $\beta\left(b_{0} \cdot b_{1}\right)=$ $\left(\Lambda\left\{x \in X: b_{0} \cdot b_{1} \leqq f x\right\}\right) \cdot \beta b_{0} \cdot \beta b_{1}$ if $S \neq \varnothing$, and $\beta\left(b_{0} \cdot b_{1}\right)=\beta b_{0} \cdot \beta b_{1}$ if $S=$ $\varnothing$. Let $a_{0}$ denote the right-hand element; that is, $a_{0}=(\Lambda S) \cdot \beta b_{0} \cdot \beta b_{1}$ if $S \neq \varnothing, a_{0}=\beta b_{0} \cdot \beta b_{1}$ if $S=\varnothing$. Clearly $f a_{0}=b_{0} \cdot b_{1}$. We show that for all $a \in F L(X)$, if $b_{0} \cdot b_{1} \leqq f a$ then $a_{0} \leqq a$. Let $T$ be the set of $a \in F L(X)$ for which this is true. Obviously $X \subseteq T$ and $T$ is closed under product. If $a, a^{\prime} \in T$ and $b_{0} \cdot b_{1} \leqq f a+f a^{\prime}$, then, by Whitman's (W2) in $L$, we have at least one of the following: $b_{0} \leqq f a+f a^{\prime}, b_{1} \leqq$ $f a+f a^{\prime}, b_{0} \cdot b_{1} \leqq f a, b_{0} \cdot b_{1} \leqq f a^{\prime} ;$ in the first case have $a+a^{\prime} \geqq \beta b_{0} \geqq a_{0}$; in the third case $a_{0} \leqq a$ by assumption $a \in T$, so $a_{0} \leqq a+a^{\prime}$; the other cases are similar, so that $a+a^{\prime} \in T$. Thus $T=F L(X)$, and $a_{0}=$ $\beta\left(b_{0} \cdot b_{1}\right)$, as desired.

Now let the endomorphism $h: F L(X) \rightarrow F L(X)$ be the extension of the map $x \rightarrow \alpha f x, x \in X$. We claim that for each $a \in F L(X), \beta f a \leqq$ $h a \leqq \alpha f a$ (so that $f h a=f a$ ). The property is obvious for $x \in X$. Proceeding inductively, suppose $\beta f a_{i} \leqq h a_{i} \leqq \alpha f a_{i}$ for $i \in\{0,1\}$; then, using the properties of $\alpha$ and $\beta$ established above, we have $\beta f\left(a_{0}+x_{1}\right)=$ $\beta\left(f a_{0}+f a_{1}\right)=\beta f a_{0}+\beta f a_{1} \leqq h a_{0}+h a_{1}=h\left(a_{0}+a_{1}\right) \leqq \alpha f a_{0}+\alpha f a_{1} \leqq \alpha\left(f a_{0}+\right.$ $\left.f a_{1}\right)$; similarly, $\beta f\left(a_{0} \cdot a_{1}\right) \leqq h\left(a_{0} \cdot a_{1}\right) \leqq \alpha f\left(a_{0} \cdot a_{1}\right)$.

Define the map $g: L \rightarrow F L(X)$ by $g=h \circ \beta$. We show that $g$ is a homomorphism of $L$ into $F L(X)$. Since $\beta$ and $h$ preserve sum, so does $g$. Now for $b_{0}, b_{1} \in L$, we must show that $h \beta\left(b_{0} \cdot b_{1}\right)=h \beta b_{0} \cdot h \beta b_{1}$; it suffices to show $h\left(\beta b_{0} \cdot \beta b_{1}\right) \leqq h \beta\left(b_{0} \cdot b_{1}\right)$. If $\beta\left(b_{0} \cdot b_{1}\right)=\beta b_{0} \cdot \beta b_{1}$ this is trivial; thus we may assume by above that $\left\{x \in X: b_{0} \cdot b_{1} \leqq f x\right\} \neq \varnothing$ and that $h \beta\left(b_{0} \cdot b_{1}\right)=\left(\Lambda\left\{h x: x \in X\right.\right.$ and $\left.\left.b_{0} \cdot b_{1} \leqq f x\right\}\right) \cdot h \beta b_{0} \cdot h \beta b_{1}$. Therefore, it suffices to show that $h\left(\beta b_{0} \cdot \beta b_{1}\right) \leqq h x$ whenever $x \in X$ and $b_{0} \cdot b_{1} \leqq f x$. But for any such $x, f\left(\beta b_{0} \cdot \beta b_{1}\right)=f \beta b_{0} \cdot f \beta b_{1}=b_{0} \cdot b_{1} \leqq f x$, so that $\beta b_{0} \cdot \beta b_{1} \leqq \alpha f x=h x$ (see definition of $h$ ); thus, using the claim of the preceding paragraph, we have $h\left(\beta b_{0} \cdot \beta b_{1}\right) \leqq h h x \leqq \alpha f h x=\alpha f x=$ 
$h x$, as desired. Thus $g$ is a homomorphism of $L$ into $F L(X)$.

To prove $L$ projective it remains only to show that for each $b \in L, f g b=b$. In fact, $f g b=f h \beta b=f \beta b=b$, as desired (the middle equality holds by our claim concerning $h$ ). This completes the proof of 3.3 .

THEOREM 3.4. A finitely generated lattice is projective iff it is imbeddable in a free lattice.

Proof. We already know that a projective lattice is imbeddable in a free lattice. Now suppose that $L$ is a sublattice of $F L(Y), L$ finitely generated. For some sufficiently large finite $X$, there is an epimorphism $f$ of $F L(X)$ onto $L$. By Corollary 3.2, $f$, viewed as a homomorphism into $F L(Y)$, is bounded; by Note 2.4, $f$ is bounded as an epimorphism onto $L$. Thus $L \in \mathscr{B}$; and $L$ inherits Whitman's (W2) from $F L(Y)$. It follows from 3.3 that $L$ is projective.

Notice that, by Theorem 3.3 and the earlier remarks, for a lattice $L$ of $L$ is projective iff $L$ is imbeddable in a free lattice iff Whitman's (W2) holds in $L$.

We have a fair amount of information on the relationship between 2 and the class of projective lattices. Our spacific examples above include lattices which are in both classes, in neither, in one class but not the other. From [1] we have the example $2 \times \omega$, a denumerable distributive non-projective lattice imbeddable in $F L(3)$; it is now clear from Theorem 3.3 that $2 \times \omega \notin \mathscr{B}$. In [5, Figure $5 \mathrm{~B}$, p. 49] we display a denumerable non-modular sublattice of $F L(3)$ which also is non-projective and not in $\mathscr{B}$. We sketch a proof of the following additional fact:

Theorem 3.5. Suppose $L$ is a distributive lattice, $L \in \mathscr{B}$, and Whitman's (W2) holds in L. Then $L$ is a finite projective lattice.

Proof. We know that $L$ is projective by Theorem 3.3. By Lemma 2.7, $L$ is a linear sum of just finitely many linear components. The Galvin-Jónsson result mentioned earlier implies that any infinite linear component of $L$ must be isomorphic to the direct product of a countable chain and a two-element chain. An argument similar to that of Lemma 2.7 now shows that there is no infinite linear component of $L$. Thus $L$ is a finite projective lattice.

\section{REFERENCES}

1. K. A. Baker and A. W. Hales, Distributive projective lattices, Canad. J. Math., 22 (1970), 472-475. 
2. F. Galvin and B. Jónsson, Distributive sublattices of a free lattice, Canad. J. Math., 13 (1961), 265-272.

3. B. Jónsson, Sublattices of a free lattice, Canad. J. Math., 13 (1961), 256-264.

4. A. Kostinsky, Finitely generated projective lattices. Preliminary report, Notices Amer. Math. Soc., 16 (1969), 563-564.

5. - Some problems for rings and lattices within the domain of general algebra, Doctoral dissertation, University of California, Berkeley, 1969.

6. R. McKenzie, Equational bases and non-modular lattice varieties, (To appear.)

7. P. M. Whitman, Free Lattices, Ann. of Math., Ser. 2, 42 (1941), 325-330.

8. - Free Lattices II., Ann. of Math., Ser. 2, 43 (1942), 104-115.

Received August 27, 1970. The contents of this paper form part of the author's doctoral disssertation [5], written under the direction of Professor Ralph McKenzie at the University of California, Berkeley. The work was supported mainly by the National Science Foundation. This paper was revised on the basis of several comments made by the referee.

Humboldt State College 
